PROCEEDINGS OF THE

AMERICAN MATHEMATICAL SOCIETY

Volume 127, Number 9, Pages 2807-2809

S 0002-9939(99)05395-2

Article electronically published on May 19, 1999

\title{
A GEOMETRIC PROOF OF A THEOREM ABOUT NON-DUAL RENORMINGS
}

\author{
LIBOR VESELÝ
}

(Communicated by Dale Alspach)

\begin{abstract}
We give a simple geometric proof of a result by Davis and Johnson that every nonreflexive Banach space $X$ admits an equivalent norm in which $X$ is not isometric to a dual space. Moreover, our renorming keeps unchanged the original norm on a given finite-codimensional subspace and makes this subspace norm-one complemented.
\end{abstract}

Let $X$ be a real Banach space. We shall say that a norm $\|\cdot\|$ on $X$ (or the space $(X,\|\cdot\|))$ is non-dual if $(X,\|\cdot\|)$ is not isometric to a dual space.

W. J. Davis and W. B. Johnson proved in [DJ] that every nonreflexive Banach space admits a non-dual renorming. This result was strengthened by D. van Dulst and I. Singer ([vDS]), who produced a renorming of any nonreflexive space such that the renormed space is not norm-one complemented in its bidual. Finally, S. V. Konyagin gave a quite simple proof of the following yet stronger result [Ko]: every nonreflexive Banach space has an equivalent norm in which a three-point set fails to have Chebyshev centers. (See, e.g., [Ho] for the definition of Chebyshev centers and for the fact that each bounded subset of $X$ admits a Chebyshev center whenever $X$ is norm-one complemented in its bidual.)

We present here a simple short geometric proof of a stronger form of the result by Davis and Johnson (Corollary 2). Here "simple" means that it uses only classical tools (James' theorem, Krein-Šmulyan theorem) and not results from renorming theory.

For a Banach space $X$, we denote by $B_{X}$ its closed unit ball and by $X^{*}$ its dual space.

Theorem. Let $(X,\|\cdot\|)$ be a nonreflexive Banach space. Then there exists a norm $\|\cdot\|$ on $X \oplus \mathbb{R}$ such that

(a) $\|(x, 0)\|=\|x\|$;

(b) the natural projection $P: X \oplus \mathbb{R} \rightarrow X, P(x, t)=x$, has norm one;

(c) $(X \oplus \mathbb{R},\|\cdot\|)$ is non-dual.

Proof. Let $f \in X^{*}$ be any functional that does not attain its norm (cf. [Ja]). Let us denote

$$
L=B_{X} \cap f^{-1}(0), \quad C=\overline{\operatorname{conv}}\left[\left(B_{X} \times\{0\}\right) \cup(L \times\{1\}) \cup(L \times\{-1\})\right] .
$$

Received by the editors September 22, 1998 and, in revised form, November 30, 1998.

1991 Mathematics Subject Classification. Primary 46B03; Secondary 46B10.

(C)1999 American Mathematical Society 
(The reader is invited to use an easily made diagram.) Then $B_{X \oplus_{1} \mathbb{R}} \subset C \subset B_{X \oplus_{\infty} \mathbb{R}}$, and $C$ is symmetric. (The symbols $\oplus_{1}$ and $\oplus_{\infty}$ denote respectively the $\ell_{1}$-sum and the $\ell_{\infty}$-sum.) Thus $C$ is the unit ball of an equivalent norm $\|\cdot\|$ on $X \oplus \mathbb{R}$, and moreover,

$$
B_{X}=\{x \in X:(x, 0) \in C\} \subset P(C) \subset P\left(B_{X \oplus \infty} \mathbb{R}\right)=B_{X} .
$$

Thus (a), (b) hold.

To prove $(\mathrm{c})$, suppose that $(X \oplus \mathbb{R},\|\cdot\| \|)$ is isometric to $Z^{*}$. If we denote by $w^{*}$ the weak-star topology $\sigma((X \oplus \mathbb{R},\|\cdot\|), Z)$, then $C$ is $w^{*}$-compact, and hence also the set

$$
L_{0}:=L \times\{0\}=((0,1)+C) \cap((0,-1)+C)
$$

is $w^{*}$-compact. This implies that also

$$
\left(f^{-1}(0) \times \mathbb{R}\right) \cap C=L \times[-1,1]=\operatorname{conv}\left[\left((0,1)+L_{0}\right) \cup\left((0,-1)+L_{0}\right)\right]
$$

is $w^{*}$-compact. By the Krein-Šmulyan theorem (cf. [D-S] or $\left.[\mathrm{Sch}]\right), f^{-1}(0) \times \mathbb{R}$ is $w^{*}$-closed since it is $b w^{*}$-closed. But $f^{-1}(0) \times \mathbb{R}$ is the kernel of the functional $F=(f, 0) \in X^{*} \oplus \mathbb{R}=(X \oplus \mathbb{R})^{*}$. Hence $F$ can be identified with an element of $Z$, and $F$ attains its norm on $C$. In other words, $F^{-1}(\|F\|)$ intersects $C$. But this is in contradiction with the fact that $f$ does not attain its norm on $B_{X}$. Indeed, $f^{-1}(\|f\|)$ does not intersect $B_{X}$, and (by (a), (b)) $\|f\|=\|F\|$; thus $F^{-1}(\|F\|)=$ $f^{-1}(\|f\|) \times \mathbb{R}$ does not intersect $B_{X} \times \mathbb{R}$ and the latter set contains $C$.

Corollary 1. For every positive integer n, each nonreflexive Banach space is isometric to an n-codimensional norm-one complemented subspace of a non-dual Banach space.

Proof. Apply the Theorem $n$ times.

From Corollary 1, we obtain the following strengthening of the theorem by Davis and Johnson.

Corollary 2. Let $E$ be a nonreflexive Banach space and let $X \subset E$ be a proper closed subspace of finite codimension. Then $E$ has an equivalent non-dual norm which coincides with the original norm on $X$ and makes $X$ norm-one complemented.

Proof. Observe that $E$ is isomorphic with $X \oplus \mathbb{R}^{n}$ for $n=\operatorname{codim} X$ (where the norm on $X$ is the one inherited from $E$ ), and $X$ is nonreflexive. Apply Corollary 1.

\section{REFERENCES}

[DJ] W. J. DAVIS and W. B. JOHNSON, A renorming of non-reflexive Banach spaces, Proc. Amer. Math. Soc. 37 (1973), 486-488. MR 46:9693

[vDS] D. van DULST and I. SINGER, On Kadec-Klee norms on Banach spaces, Sudia Math. 54 (1976), 205-211. MR 52:14937

[D-S] N. DUNFORD and J. T. SCHWARTZ, Linear Operators I, New York 1958. MR 22:8302

[Ho] R. B. HOLMES, A course in Optimization and Best Approximation, Lecture Notes in Mathematics 257, Springer-Verlag, 1972. MR 54:8381

[Ja] R. C. JAMES, Reflexivity and the supremum of linear functionals, Ann. Math. 66 (1957), 159-169. MR 19:755g 
[Ko] S. V. KONYAGIN, A remark on renormings of nonreflexive spaces and the existence of a Chebyshev center, Moscow Univ. Math. Bull. 43, no. 2 (1988), 55-56. MR 89g:46033

[Sch] H. H. SCHAEFER, Topological Vector Spaces, Graduate Texts in Mathematics, Vol. 3, Springer-Verlag, Berlin 1971. MR 49:7722

Dipartimento di Matematica, Università degli Studi di Milano, Via C. Saldini 50, 20133 Milano, Italy

E-mail address: libor@vmimat.mat.unimi.it 